\section{Relação entre a atividade física regular e a agregação de fatores de risco biológicos das doenças cardiovasculares em crianças e adolescentes}

\section{Relationship between regular physical activity and aggregated biological risk factors in cardiovascular diseases in children and adolescents}

Sandra Guerra 1

José Oliveira 2

José Carlos Ribeiro 3

Armando Teixeira-Pinto 4

José Alberto Duarte 5

Jorge Mota 6

1-3,5,6 Gabinete de Desporto de Recreação e Tempos livres. Centro de Investigação em Atividade Física, Saúde e Lazer. Faculdade de Ciências do Desporto e de Educação Física. Universidade do Porto. Rua Dr. Plácido Costa 91. 4200450 Porto, Portugal

4 Departamento Bioestatística e Informática Médica. Universidade de Medicina. Universidade do Porto, Portugal.

\begin{abstract}
This paper analyses literature related to biological risk factors aggregation and the hypothetical relationship between physical activity (PA) and aggregation. The regular practice of physical activities has been associated to a healthy lifestyle. When risk factors stemming from cardiovascular diseases (CVD) are present during childhood and adolescence and because the level of habitual PA seem to influence these factors, more intensive PA during this period of life may have an impact in reducing morbidity and mortality of $C V D$ in adult life. Literature does not indicate biological risk factors aggregation for CVD in boys and girls, nevertheless, there is no consensus determining the highest aggregation risk for each one of the sexes. Therefore, results seem to suggest that children or adolescents identified as bearing risk factors should be followed-up to determine whether they possess more aggregated risk factors. Literature is not unanimous in relation to $P A$ and biological risk factors aggregaton for CVD.
\end{abstract}

Key words Risk factors, Cardiovascular diseases, Motor activity
Resumo Com o presente trabalho pretende-se analisar os estudos referenciados na literatura que abordam o tema da agregação de fatores de risco biológi$\cos$ e ainda analisar a hipotética relação entre a atividade física (AF) e essa agregação. O hábito de prática regular de AF tem sido associado a um estilo de vida saudável. Uma vez que os fatores de risco das doenças cardiovasculares (DCV) estão já presentes na infância e adolescência e porque os niveis de AF habitual parecem exercer alguma influência nesses fatores, indices mais elevados de AF nesses periodos de vida poderão repercutir-se na redução da morbidade e mortalidade das DCV em idades adultas. Através da análise da literatura parece existir agregação de fatores de risco biológicos para as DCV, para rapazes e moças, contudo não existe ainda consenso quanto ao sexo que registra o "risco" mais elevado de agregação. Deste modo, os resultados parecem sugerir que as crianças ou adolescentes identificados com um fator de risco, devem efetuar um rastreio no sentido de verificar se possuem mais fatores de risco agregados. De acordo com os estudos referenciados na literatura não existe unanimidade quanto à relação entre a AF e a agregação de fatores de risco biológicos para as $D C V$.

Palavras-chave Fatores de risco, Doenças cardiovasculares, Atividade motora 


\section{Atividade física crianças e adolescentes}

A atividade física (AF) habitual tem sido reconhecida como uma componente importante de um estilo de vida saudável. ${ }^{1}$ São várias as razões, sugeridas por Blair et al., ${ }^{2}$ para explicar a possível relação causal entre a AF e a saúde em crianças e adolescentes: a) as crianças com baixos índices de AF parecem ser mais susceptíveis para desenvolverem patologias degenerativas em idade adulta; b) a AF nas crianças parece induzir alterações biomecânicas, fisiológicas e psicológicas, as quais se manifestam como adaptações crônicas benéficas, persistindo de forma vantajosa durante a vida adulta; c) os hábitos da prática das atividades físicas adquiridos na infância parecem persistir durante a vida adulta. Na infância, a AF parece, assim, ter um papel importante para a estabilidade dos hábitos de prática dessas atividades, os quais poderão adquirir uma importância vital para a prevenção dos fatores de risco das doenças cardiovasculares (DCV). 3 A reforçar esta hipótese, vários estudos sugerem que os níveis de $\mathrm{AF}$ durante a vida adulta estão diretamente relacionados com o estado de saúde no adulto.4-8 É igualmente reconhecido que o nível de saúde em crianças e adolescentes pode ser um preditor importante do nível de saúde em idades adultas. $9-11$

\section{Definição de agregação e classificação de fatores de risco}

A agregação de fatores de risco de DCV é definida pela coexistência de vários fatores no mesmo sujeito. ${ }^{12} \mathrm{Na}$ literatura científica é freqüente o uso de uma classificação dos fatores de risco em função da sua natureza. A natureza biológica (ex: níveis de lipoproteínas, tensão arterial, gordura corporal, aptidão cardiopulmonar, etc) e a natureza comportamental e/ou de estilo de vida (atividade física diária, consumo de energia, tabaco, consumo de álcool, etc) são os critérios usados para distinguir e agrupar os fatores de risco.13-15

Tomando em consideração a classificação acima referida, têm sido conduzidos alguns estudos com o objetivo de determinar a influência recíproca de fatores da mesma natureza e, também, observar a interação entre os fatores de natureza biológica com os de estilo de vida e vice-versa.

\section{Agregação de fatores de risco biológicos}

Apesar de um único fator de risco poder contribuir, por si só, para o desenvolvimento de DCV, os fatores de risco biológicos tendem a agregar-se, potenciando a geração de um efeito de multiplicação de risco de desenvolvimento das referidas doenças. ${ }^{9,12,15,16-}$ $22 \mathrm{Um}$ bom exemplo de agregação de fatores de risco é a "síndrome metabólica" que se caracteriza pela coexistência de "obesidade abdominal", hipertrigliceridemia, dislipidemia, hipertensão arterial (HA) e hiperinsulinemia.23

Habitualmente são três as razões subjacentes à seleção de fatores de risco biológicos: a) serem reconhecidos como alguns dos mais importantes fatores de risco independentes para as DCV; b) o insuficiente conhecimento do poder de agregação dos fatores de risco e sua manifestação em crianças e adolescentes; c) serem considerados importantes preditores das DCV na vida adulta, podendo, assim, antecipar programas de intervenção com objetivos preventivos. 14

Berenson et al. 9 estudaram a influência da agregação de fatores de risco biológicos na extensão da aterosclerose coronária e aórtica. Para tal, realizaram um estudo numa amostra de 209 sujeitos com idades compreendidas entre dois e 39 anos de idade. Nesse estudo, o índice de massa corporal, tensão arterial sistólica, pressão arterial diastólica (PAD), colesterol total (CT), triglicerídeos, lipoproteínas de baixa densidade e as lipoproteínas de alta densidade (HDL-C), foram fortemente associados à extensão das lesões da artéria aorta e artérias coronárias. As conclusões desse estudo referem que quanto maior o número de fatores de risco biológicos agregados, maior a severidade assintomática da aterosclerose coronária e aortica. Essas conclusões reforçam também a necessidade de se investigar não só os fatores de risco das DCV de forma isolada mas, igualmente, tomando em consideração a sua agregação. ${ }^{9}$ Em reforço das conclusões do estudo de Berenson et al., 9 Haffner24 referiu que a morbidade relacionada com as DCV era duas vezes maior em sujeitos com a síndrome metabólica, comparativamente aos que não apresentavam nenhum fator de risco. De fato, várias investigações 25-28 sugerem que cerca de metade dos sujeitos estudados apresentam pelo menos um fator de risco biológico para as DCV. Estes resultados estão de acordo com um estudo recente realizado em Portugal com crianças e adolescentes de ambos os sexos, com idades compreendidas entre os oito e os 16 anos de idade. 29

Do acima exposto, considerando os estudos supra-citados, poderemos desde já salientar que, independentemente das diferenças amostrais, das variáveis selecionadas e dos procedimentos e critérios de análise, uma larga percentagem dos sujeitos submeti- 
dos a estudo apresentam pelo menos um fator de risco para as DCV. Este fato contraria uma crença mais ou menos generalizada de que, nas crianças e adolescentes, as populações estarão à margem de qualquer tipo de risco para a saúde. Assim sendo, torna-se vital o desenvolvimento de políticas e de programas de saúde pública a partir destas idades nas vertentes da educação, do acompanhamento clínico e de programas de prevenção. Por exemplo, um incremento dos níveis de AF e de dispêndio energético poderão ser um dos primeiros objetivos dos programas de prevenção.

Na literatura é referida uma agregação de fatores de risco biológicos para rapazes e moças. De fato, vários estudos demonstraram que os fatores de risco das DCV tendem a agregar-se mesmo em crianças e adolescentes. 14,15,20-22,28,30-32 Contudo ainda não existe um consenso relativamente ao sexo que apresenta um maior grau de agregação. No estudo de Twisk et al.14 a agregação dos rapazes foi ligeiramente mais elevado comparativamente à das moças. Idênticos resultados foram encontrados noutros estudos em crianças e adolescentes. ${ }^{21,32}$ Twisk et al. 14 referem que não estão ainda determinadas as razões para o grau mais elevado de agregação no sexo masculino. Twisk et al.,15 referiram não serem observadas diferenças no grau de agregação de fatores de risco biológicos entre os sexos. Contrariamente aos estudos supracitados, o estudo realizado por Guerra et al. ${ }^{29}$ parece demonstrar que a agregação de dois ou mais fatores de risco biológicos é superior nas moças.

\section{Agregação de fatores de risco de estilo de vida e/ou comportamental}

No que diz respeito aos fatores de risco de natureza comportamental e/ou de estilo de vida, é recorrentemente referida a sua manifestação de forma agregada. Se a AF tem sido associada a comportamentos ou estilos de vida saudáveis, em contrapartida, a ausência de AF aparece, muitas vezes, associada a outros fatores que aumentam a probabilidade do risco das DCV. 33 Porém, a comprovação das tendências acima referidas não está claramente estabelecida. Do nosso conhecimento, a investigação realizada até ao momento apresenta conclusões contraditórias. Como exemplo, em Raitakari et al. 34 a inatividade física associou-se ao consumo de tabaco e de álcool e ainda a uma dieta com uma composição excessiva de lípidos. Num outro estudo, realizado em 301 rapazes e 282 moças australianas com 18 anos de idade, o consumo de tabaco e de álcool, assim como hábitos ali- mentares não saudáveis, foram relacionados entre si; para as moças um nível baixo de AF foi associado a outros comportamentos não saudáveis. ${ }^{35}$ Por outro lado, para Kilkens et al.,13 a inatividade física não foi associada a qualquer outro fator de estilo de vida não saudável.

\section{Relação entre fatores de risco de estilo de vida e/ou comportamental e fatores de risco biológicos}

Em populações adultas 36,37 e também em crianças e adolescentes 14,15,21 tem sido realizada alguma investigação procurando determinar a interação entre fatores de estilo de vida e fatores biológicos. Os estudos em populações pediátricas têm apresentado resultados contraditórios no que diz respeito à hipotética influência da AF para a agregação de fatores de risco biológicos. A diferença no sentido dos resultados poder-se-á ficar a dever às diferenças de metodologia na avaliação da AF e aos diferentes fatores de risco biológicos considerados. Num estudo longitudinal integrado de Kilkens et al.,13 tendo como objetivos investigar: a agregação de fatores de estilo de vida (inatividade física, tabagismo, ingestão de álcool e consumo de dieta não favorável) e a influência dessa agregação nos fatores de natureza biológica (massa gorda, distribuição da massa gorda, aptidão cardiorrespiratória, tensão arterial, CT e razão CT/HDL-C), foram repetidas seis avaliações durante um período de 15 anos em 181 sujeitos, a partir dos 13 anos de idade. A análise da regressão linear demonstrou não existir uma relação significativa entre o score de associação (soma dos fatores de risco de estilo de vida) e os fatores de risco biológicos das DCV, com exceção para a relação inversa com a aptidão cardiorrespiratória.

Estes resultados contrastam com os encontrados no estudo de Raitakari et al. ${ }^{34}$ Nesse estudo, a dieta, o tabagismo, a inatividade física e ingestão de álcool, foram os fatores de estilo de vida, associados aos fatores biológicos. Fazendo parte do "Amsterdam Growth and Health Study" (AGHS), citado em Kilkens et al.,13 sugeriram algumas explicações para as diferenças encontradas no seu estudo relativamente aos de Raitakari et al. ${ }^{34}$ Em primeiro lugar, a dimensão da amostra de Kilkens et al.,13 o AGHS (n = 181) era menor, comparativamente à de Raitakari et al.34 o "Cardiovascular Risk in Young Finns Study” (CRYFS)34 ( $=484)$, o que tornava difícil a detecção de uma relação significativa entre os fatores. Em segundo lugar, a idade dos sujeitos nos dois estudos não era sobreponível. De fato, para 
Kilkens et al.,13 o AGHS, os sujeitos foram seguidos desde os 13 aos 27 anos de idade, enquanto no CRYFS os sujeitos foram seguidos desde os 18 aos 24 anos de idade. É possível que os sujeitos mais novos sofram uma maior influência parental comparativamente aos sujeitos mais velhos. ${ }^{13}$ Em terceiro lugar, as diferenças poderão também ser explicadas pelo nível socioeconômico e educacional dos sujeitos. Kok et al. 37 concluíram que nos sujeitos com nível educacional baixo, a tendência para a agregação de fatores de estilo de vida era duas vezes maior do que a observada em sujeitos com nível superior de educação. Da mesma forma, Raitakari et al.34 observaram que um nível superior de educação era um fator protetor da associação de fatores de risco de estilo de vida. Os sujeitos pertencentes ao AGHS eram estudantes freqüentando a mesma escola, com um nível socioeconômico superior à média das famílias holandesas. 38 Assim, a ausência de uma agregação significativa entre os fatores de risco para o desenvolvimento das DCV no AGHS poderá estar relacionada com níveis socioeconômicos e educacionais elevados. 13

Por outro lado, Twisk et al.14 no "Northern Ireland Young Hearts Project" (NIYHP), sendo um estudo realizado numa amostra de sujeitos de 12 e 15 anos de idade, não observaram qualquer tipo de relação entre a AF diária e os scores de agregação baseados na razão CT/HDL-C, na TAD, na soma de quatro pregas cutâneas e na performance obtida num teste de avaliação da aptidão cardiorrespiratória (número de percursos no teste de Shuttle Run.39 Deste modo, através da análise da literatura, a influência da AF para a agregação de fatores de risco biológicos não está bem determinada. Contudo, é necessário realçar que nos estudos supracitados, é considerada uma diferente combinação de fatores de risco biológicos, a dimensão e as idades da população estudada são diferentes assim como são também diferentes os procedimentos de análise dos dados e a metodologia para avaliar a AF.

De um ponto de vista preventivo, é especialmente importante investigar que fatores de estilo de vida se relacionam com a agregação de fatores de risco biológicos das DCV, elegendo-os, à posteriori, como alvos das estratégias de prevenção. 40 Sem dúvida que uma importante estratégia primária de prevenção das DCV é a alteração dos fatores de estilo de vida tal como o incremento da AF, eliminação de hábitos tabágicos e a adoção de hábitos alimentares saudáveis.15,41,43 Estas alterações são importantes, não só porque os fatores de estilo de vida se relacionam diretamente com as DCV, mas também porque se relacionam indiretamente com os fatores de risco biológicos como a hipercolesterolemia, a HA e a obesidade. 44

Ressaltamos que se torna difícil comparar os nossos resultados com a literatura, relativamente à agregação de fatores de risco biológicos e, ainda, à relação entre os fatores de estilo de vida e a agregação de fatores de risco biológicos para as DCV. Esta situação poderá resultar do fato de, noutros estudos, os fatores de risco e a sua combinação serem diferentes. Por exemplo, em vários trabalhos, a hiperinsulinemia foi considerada na agregação de fatores de risco biológicos. ${ }^{45-48}$ Contudo, no nosso estudo 48 e em outros, 15,49 a hiperinsulinemia não foi considerada. Desta forma, como nos diversos estudos se efetuaram diferentes associações, quer de fatores de risco biológicos quer de fatores de estilo de vida, a comparação dos vários resultados entre os trabalhos torna-se difícil.

O estudo de Suter e Hawes 50 refere a associação entre a AF, a composição corporal e o perfil lipídico sanguíneo. Ou seja, uma relação positiva entre a AF e as HDL-C e uma relação negativa entre a soma das pregas cutâneas e a razão CT/HDL-C. Estes resultados foram similares aos encontrados noutros estudos, 51-53 nos quais o perfil lipídico das crianças ativas era mais favorável comparativamente às das crianças sedentárias. Um outro estudo, concluiu que a AF se encontrava associada a um perfil lipídico favorável na infância e em idades adultas jovens, particularmente no sexo masculino. 46

Twisk et al. 49 apontam uma razão para não se terem registrado diferenças significativas entre os scores de agregação dos fatores de risco biológicos e os fatores de estilo de vida. Esta razão está intimamente relacionada com a definição de pontos de corte estabelecidos para definir as crianças e adolescentes em risco das DCV. Isto é, a adoção de valores de risco relativos, de que são exemplo os quartis, em vez de valores absolutos de risco. Assim, é possível que um sujeito no percentil 75 para um fator de risco não possua valores absolutos elevados. Por exemplo, no estudo de Twisk et al. 49 a média da PAD para o quarto quartil das crianças com 12 anos de idade foi de $80.0 \mathrm{mmHg}$ para os rapazes e de $82,6 \mathrm{mmHg}$ para as moças. Para os sujeitos quando tinham 15 anos de idade, o quarto quartil ainda se apresentou mais baixo $(77,7 \mathrm{mmHg}$ para rapazes e $77,9 \mathrm{mmHg}$ para moças). Estes valores são inferiores aos valores de PAD dos sujeitos com idades similares. 54

No estudo de Guerra et al.48 é importante referir que pela análise da agregação de fatores de risco biológicos nos dois sexos, a quantidade de AF necessária para contrariar a agregação de dois ou mais fatores de risco será menor no sexo feminino 
comparativamente ao masculino, o que pode ser um importante indicador para projetos de intervenção.

Se compararmos a variação dos valores de agregação do primeiro para os segundo e terceiro quartis do IAF, verificamos que no sexo feminino o decréscimo é relativamente acentuado enquanto no masculino não se regista grande alteração. Deste modo, os nossos resultados sugerem que uma quantidade relativamente pequena de AF parece influenciar favoravelmente o perfil de risco das DCV, fundamentalmente no sexo feminino.

\section{Agradecimentos}

Este estudo foi financiado pela Fundação para a Ciência e Tecnologia (FCT), Programa Praxis XXI: PSAU/122/96.

\section{Referências}

1. Twisk JW. Physical activity guidelines for children and adolescents: a critical review. Sports Med 2001; 31: 617-27.

2. Blair S, Clark D, Cureton K, Powell K. Exercise and finess in childhood: implications for a lifetime of health. In: Gisolfi, CV, Lam, DR. Perspectives in exercise science and sports medicine. 2 ed. Indianapolis: Benchmark; 1989. p. 401-30.

3. Sharp N. Activity of children-health through sport, exercise and fitness? In: Chan KM, Micheli LJ. Sport and children. Champaign, IL: Human kinetics; 1998. p. 39-49.

4. Lee IM, Paffenbarger RSJr, Hsieh C. Physical activity and risk of developing colorectal cancer among college Alumni. J Natl Cancer Inst 1991; 83: 1324-9.

5. Leon AS, Connett J, Jacobs DRJr, Rauramaa R. Leisure-time physical activity levels and risk of coronary heart disease and death. The multiple risk factor intervention trial. JAMA 1987; 258: 2388-95.

6. McClaran SR, Babcock MA, Pegelow DF, Reddan WG, Dempsey JA. Longitudinal effects of aging on lung function at rest and exercise in healthy active fit elderly adults. J Appl Physiol 1995; 78: 1957-68.

7. Powell KE, Blair SN. The public health burdens of sedentary

\section{Conclusões}

Através da análise da literatura parece existir agregação de fatores de risco biológicos para as DCV, para rapazes e moças, contudo parece não existir consenso quanto ao sexo que registra o grau mais elevado de agregação. Deste modo, os resultados sugerem que crianças ou adolescentes identificados com um fator de risco, devem ser rastreados no sentido de verificar se possuem mais fatores de risco agregados. De acordo com os estudos referenciados na literatura não existe unanimidade quanto à influência da $\mathrm{AF}$ na agregação de fatores de risco biológicos para as DCV. living habits: theoretical but realistic estimates. Med Sci Sports Exerc 1994; 26: 851-6.

8. Taylor CB, Sallis JF, Needle R. The relation of physical activity and exercise to mental health. Public Health Rep 1985; 100: 195-202.

9. Berenson GS, Srinivasan SR, Bao W, Newman WP, Tracy III RE, Wattigney WA. Association between multiple cardiovascular risk factors and atherosclerosis in children and young adults: the Bogalusa heart study. N Engl J Med 1998; 338: 1650-6.

10. Strong WB, Deckelbaum RJ, Gidding SS, Kavey RE, Washington R, Wilmore JH, Perry CL. Integrated cardiovascular health promotion in childhood: a statement for health professionals from the Subcommittee on Atherosclerosis and Hypertension in Childhood of the Council on Cardiovascular Disease in the Young. Circulation 1992; 85: 1638-50

11. Twisk JW, Kemper HC, Van Mechelen W, Post GB. Tracking of risk factors for coronary heart disease over a 14-year period: a comparison between lifestyle and biologic risk factors with data from the Amsterdam Growth and Health Study. Am J Epidemiol 1997; 145: 888-98. 
12. Twisk J. Physical activity, physical fitness and cardiovascular health. In: Armstrong N, Van Mechelen, W. Paediatric exercise science and medicine. Oxford: Oxford University; 2000. p. 253-63.

13. Kilkens O, Gijtenbeek B, Twisk J, Van Mechelen W, Kemper C. Clustering of lifestyle CVD risk factors and its relationship with biological CVD risk factors. Pediatr Exerc Sci 1999; 11: 169-77.

14. Twisk JW, Boreham C, Cran G, Savage JM, Strain J, Van Mechelen W. Clustering of biological risk factors for cardiovascular disease and the longitudinal relationship with lifestyle of an adolescent population: the Northern Ireland Young Hearts Project. J Cardiovasc Risk 1999; 6: 355-62.

15. Twisk JW, Kemper HC, Van Mechelen W, Post GB. Clustering of risk factors for coronary heart disease. The longitudinal relationship with lifestyle. Ann Epidemiol 2001; 11: 157-65.

16. Bao W, Srinivasan SR, Wattigney WA, Berenson GS. Persistence of multiple cardiovascular risk clustering related to syndrome $\mathrm{x}$ from childhood to young adulthood: the Bogalusa heart study. Arch Intern Med 1994; 154: 1842 7.

17. Genest JJr, Cohn JS. Clustering of cardiovascular risk factors: targeting high-risk individuals. Am J Cardiol 1995; 76: $8 \mathrm{~A}-20 \mathrm{~A}$

18. Genest JJ, McNamara JR, Salem DN, Schaefer EJ. Prevalence of risk factors in men with premature coronary artery disease. Am J Cardiol 1991; 67: 1185-9.

19. Jousilahti P, Toumilehto J, Vartiainen E, Korhonen HJ, Pitkaniemi J, Nissinen A, Puska P. Importance of risk factor clustering in coronary heart disease mortality and incidence in Eastern Finland. J Cardiovasc Risk 1995; 2: 63-70.

20. Khoury P, Morrison JA, Kelly K, Mellies M, Horvitz R, Glueck CJ. Clustering and interrelationships of coronary heart disease risk factors in schoolchildren, ages 6-19. Am J Epidemiol 1980; 112: 524-38.

21. Raitakari OT, Porkka KV, Rasanen L, Ronnemaa T, Viikari JS. Clustering and six year cluster-tracking of serum total cholesterol, HDL-cholesterol and diastolic blood pressure in children and young adults. the cardiovascular risk in young finns study. J Clin Epidemiol 1994; 47: 1085-93.

22. Smoak CG, Burke GL, Webber LS, Harsha DW, Srinivasan SR, Berenson GS. Relation of obesity to clustering of cardiovascular disease risk factors in children and young adults: the Bogalusa heart study. Am J Epidemiol 1987; 125: 364-72.

23. Reaven G. Role of insulin resistance in human disease. Diabetes 1988; 37: 1595-607.

24. Haffner SM. Epidemiology of insulin resistance and its relation to coronary artery disease. Am J Cardiol 1999; 84 $11 \mathrm{~J}-4 \mathrm{~J}$.

25. Berenson A. Cardiovascular risk factors in children: the early natural history of atherosclerosis and essencial hypertension. New York: Oxford University; 1980.

26. Boreham C, Savage JM, Primrose D, Cran G, Strain J. Coronary risk factors in schoolchildren. Arch Dis Child 1993; 68: 182-6.

27. Bouziotas C, Koutedakis Y, Shiner R, Pananakakis Y, Fotopoulou V, Gara S. The prevalence of selected modifiable coronary heart disease risk factors in 12-year-old
Greek boys and girls. Pediatr Exerc Sci 2001; 13: 17384.

28. Grunberg H, Thetloff M. The cardiovascular risk factor profile of Estonian school children. Acta Paediatr 1998; 87: 37-42.

29. Guerra S, Oliveira J, Ribeiro J, Teixeira-Pinto A, Twisk JWR, Duarte J, Mota J. Physical activity and biological risk factors clustering in Portuguese children. Int $\mathrm{J}$ Sports Med 2002. [in press].

30. Boulton TJ, Johnston O. A coronary risk-factor profile of 4 year olds. II. Inter-relationships, clustering, and tracking of blood pressure, serum lipoproteins, and skinfold thickness. Aust Paediatr J 1978; 14: 278-82.

31. Lauer RM, Connor WE, Leaverton PE, Reiter MA, Clarke WR. Coronary heart disease risk factors in school children: the muscatine study. J Pediatr 1975; 86: 697-706.

32. Webber LS, Voors AW, Srinivasan SR, Frerichs RR, Berenson GS. Occurrence in children of multiple risk factors for coronary artery disease: the Bogalusa heart study. Prev Med 1979; 8: 407-18.

33. Simons-Morton BG, O'Hara NM, Parcel GS, Huang IW, Baranowski T, Wilson B. Children's frequency of participation in moderate to vigorous physical activities. Res Q Exerc Sport 1990; 61: 307-14.

34. Raitakari OT, Leino M, Rakkonen K, Porkka KV, Taimela S, Rasanen L, Viikari JS. Clustering of risk habits in young adults. The cardiovascular risk in young finns study. Am J Epidemiol 1995; 142: 36-44.

35. Burke V, Milligan RA, Beilin LJ, Dunbar D, Spencer M, Balde E, Gracey MP. Clustering of health-related behaviors among 18-year-old Australians. Prev Med 1997; 26: 724-33.

36. Criqui MH, Barrett-Connor E, Holdbrook MJ, Austin M, Turner JD. Clustering of cardiovascular disease risk factors. Prev Med 1980; 9: 525-33.

37. Kok FJ, Matroos AW, Van Den Ban AW, Hautvast JG. Characteristics of individuals with multiple behavioral risk factors for coronary heart disease: the Netherlands. Am J Public Health 1982; 72: 986-91.

38. Kemper H. A longitudinal analysis of health, fitness and lifestyle: Amsterdam growth and health study. Champaign, IL: Human kinetics; 1995. (HK Sport Science Monograph Series)

39. Durnin JV, Lonergan ME, Good J, Ewan A. A cross-sectional nutritional and anthropometric study, with an interval of 7 years, on 611 young adolescent schoolchildren. Br J Nutr 1974; 32: 169-79.

40. Kilkens O, Gijtenbeek B, Twisk J, Van Mechelen W, Kemper C. Clustering of lifestyle CVD risk factors and its relationship with biological CVD risk factors. Pediatr Exerc Sci 1999; 11: 169-77.

41. Superko HR, Haskell WH. The role of exercise training in the therapy of hyperlipoproteinemia. Cardiol Clin 1987; 5: 285-310.

42. Wood PD, Stefanick ML, Williams PT, Haskell WL. The effects on plasma lipoproteins of a prudent weight-reducing diet, with or without exercise, in overweight men and women. N Engl J Med 1991; 325: 461-6.

43. Hubert HB, Eaker ED, Garrison RJ, Castelli WP. Life-style correlates of risk actor change in young adults: an eightyear study of coronary heart disease risk factors in the framingham offspring. Am J Epidemiol 1987; 125: 81231 . 
44. Bao W, Srinivasan SR, Wattigney WA, Berenson GS. Persistence of multiple cardiovascular risk clustering related to syndrome $\mathrm{x}$ from childhood to young adulthood: the Bogalusa heart study. Arch Intern Med 1994; 154: 18427.

45. Hovell M, Sallis J, Kolody B, McKenzie T. Children's physical activity choices: a development analysis of gender, intensity levels, and time. Pediar Exerc Sci 1999; 11: 158-68.

46. Raitakari OT, Taimela S, Porkka KV, Telama R, Valimaki I, Akerblom HK, Viikari JS. Associations between physical activity and risk factors for coronary heart disease: the cardiovascular risk in young finns study. Med Sci Sports Exerc 1997; 29: 1055-61.

47. Whaley MH, Kampert JB, Kohl III HW, Blair SN. Physical fitness and clustering of risk factors associated with the metabolic syndrome. Med Sci Sports Exerc 1999; 31: 287-93.

48. Guerra S, Oliveira J, Ribeiro J, Teixeira-Pinto A, Twisk JWR, Duarte J, Mota J. Physical activity and biological risk factors clustering in Portuguese children. Int $\mathbf{J}$ Sports Med 2002. [in press].

Recebido em 10 julho de 2002

Versão final reapresentada em 20 setembro de 2002

Aprovado em 10 novembro de 2002
49. Twisk JW, Boreham C, Cran G, Savage JM, Strain J, Van Mechelen W. Clustering of biological risk factors for cardiovascular disease and the longitudinal relationship with lifestyle of an adolescent population: the Northern Ireland young hearts project. J Cardiovasc Risk 1999; 6: 355-62.

50. Suter E, Hawes MR. Relationship of physical activity, body fat, diet, and blood lipid profile in youths 10-15 yr. Med Sci Sports Exerc 1993; 25: 748-54.

51. Kemper HC, Snel J, Verschuur R, Storm-van Essen L. Tracking of health and risk indicators of cardiovascular diseases from teenager to adult: Amsterdam growth and health study. Prev Med 1990; 19: 642-55.

52. Parizkova J, Mackova E, Kabele J, Mackova J, Skopkova M. Body composition, food intake, cardirespiratory fitness, blood lipids and psychological development in highly active and inactive preschool children. Hum Biol 1986; 58: 261-73.

53. Thorland WG, Gilliam TB. Comparison of serum lipids between habitually high and Low active pre-adolescent males. Med Sci Sports Exerc 1981; 13: 316-21.

54. Report of the second task force on blood pressure control in children. Pediatrics 1987; 79: 1-25. 